\title{
OPEN The value of three narrow-band imaging model in the diagnosis of small colorectal polyps
}

\author{
Jie Sha ${ }^{1,6}$, Pin Wang ${ }^{2,6}$, Nan Sang ${ }^{3,6}$, Huiai Zhang ${ }^{4,6}$, Aiping Yang ${ }^{1}$, Lei Chen ${ }^{1}$, Zewei Gong ${ }^{1}$, \\ Chao $\mathrm{Li}^{1}$, Yumeng Qin ${ }^{1}$, Xueliang $\mathrm{Li}^{5}$, Yong $\mathrm{Ji}^{1 \bowtie}$ \& Feng Gao ${ }^{1 \bowtie}$
}

This prospective study was aimed to evaluate the clinical value of narrow-band imaging (NBI), magnification endoscopy with narrow-band imaging (NBIME) and magnification endoscopy with acetic acid enhancement and narrow-band imaging (AA-NBIME) in the diagnosis of small colorectal polyps. We studied 261 small colorectal polyps from 122 patients with the use of above three techniques. Lesions were resected for histopathological analysis. The endoscopic images were independently reviewed by three experts and three non-experts and the diagnostic accuracy and image definition were compared among the modalities. The "experts-agreed" diagnostic accuracy was $\mathbf{8 7 . 7 \%}$ for NBI versus $91.6 \%$ for NBIME versus $94.6 \%$ for AA-NBIME. The "non-experts-agreed" diagnostic accuracy was $\mathbf{8 0 . 1 \%}$ for NBI versus $\mathbf{8 4 . 3 \%}$ for NBIME versus $\mathbf{8 9 . 3 \%}$ for AA-NBIME. All experts and non-experts diagnosed the small colorectal polyps statistically more accurately with AA-NBIME than NBI $(P<0.05)$. In all three modalities, the expert group's diagnostic accuracies were statistically significantly higher compared with the non-expert group. For experts, the Kappa values for AA-NBIME, NBIME and NBI diagnosis were 0.962 (0.892-1.032), $0.577(0.507-0.647)$ and 0.567 (0.497-0.637), respectively; while for nonexperts, $0.818(0.748-0.888), 0.532(0.462-0.602)$ and 0.530 $(0.460-0.600)$. This demonstrated a good reproducibility of AA-NBIME diagnosis. The average scores (experts and non-experts) of images acquired using AA-NBIME were significantly higher than those acquired using NBIME and ME $(P<0.05)$. AA-NBIME is a promising tool to clearly visualize the mucosal pit pattern (PP) of colorectal polyps for better differentiating neoplastic polyps from non-neoplastic ones.

Colorectal cancer (CRC) is the third most common cancer and the fourth leading cause of cancer death in the world ${ }^{1}$. Nowadays it is generally accepted that adenomatous polyps (AP) are precursors of CRC ${ }^{2}$, and their removal significantly reduces the incidence of $\mathrm{CRC}^{3,4}$. However, the conventional colonoscopy is not enough to distinguish non-neoplastic from neoplastic polyps. Hence, a considerable proportion of polyps are proved to be non-neoplastic according to histopathological assessment and they are unnecessary to be resected and sent for histopathological analysis, which may add to the potential cost and anxiety of patients due to the delay of pathology results. Differentiating neoplastic from non-neoplastic polyps in a more effective way is essential for determining appropriate treatment.

Much effort has been made on the new technologies to improve diagnostic accuracy to differentiate neoplastic from non-neoplastic colorectal lesions. NBI is a technology that uses 3 optical filters for red-blue-green sequential illumination and narrows the bandwidth of the spectral transmittance supporting the imaging of mucosal pit patterns (PPs) and capillary vessels ${ }^{5}$. In 2012, NBI International Colorectal Endoscopic (NICE) classification using NBI was developed and also validated internationally applicable in diagnosing colorectal polyps ${ }^{6}$. Magnification endoscopy combined with NBI (NBIME) has been reported to better show the details of mucosal PPs and capillaries; therefore, this technique has become useful to distinguish neoplastic from non-neoplastic lesions according to the NICE classification. However, in some cases, it is hard to interpret the findings of $\mathrm{NBIME}^{7,8}$,

\footnotetext{
${ }^{1}$ Department of Gastroenterology, Jingjiang People's Hospital, Jingjiang 214500, Jiangsu, China. ${ }^{2}$ Department of Gastroenterology, Nanjing Drum Tower Hospital, Affiliated Hospital of Nanjing University Medical School, Nanjing 210008, Jiangsu, China. ${ }^{3}$ Department of Gastroenterology, The Geriatric Hospital of Nanjing Medical University, Nanjing 210024, Jiangsu, China. "'Department of Gastroenterology, Taixing People's Hospital, Taizhou 225400, Jiangsu, China. ${ }^{5}$ Department of Gastroenterology, The First Affiliated Hospital of Nanjing Medical University, Nanjing 210029, Jiangsu, China. ${ }^{6}$ These authors contributed equally: Jie Sha, Pin Wang, Nan Sang and Huiai Zhang. ${ }^{\circledR}$ email: 42394176@qq.com; 1036060339@qq.com
} 


\begin{tabular}{|l|l|}
\hline Patients $(\mathrm{n})$ & 122 \\
\hline Age (years) mean \pm SD & $54.2 \pm 11.8$ \\
\hline Male & 85 \\
\hline Female & 37 \\
\hline Polyps $(\mathrm{n})$ & 261 \\
\hline $1-5 \mathrm{~mm}$ & 132 \\
\hline $6-10 \mathrm{~mm}$ & 129 \\
\hline Right colon & 79 \\
\hline Left colon & 119 \\
\hline Rectum & 63 \\
\hline
\end{tabular}

Table 1. Clinical characteristics.

and is not known as to the potential advantage of NBIME over NBI in the histological differential diagnosis of Small colorectal polyps.

Magnification endoscopy with acetic acid-enhanced NBI (AA-NBIME) is considered to be an effective method for observing the microstructure of the mucosal surface ${ }^{9}$. This modality can vividly observe the deep brown glandular epithelial crypts and the whitish area between the crypts. This whitish area is a reversible molecular structure change of cellular proteins induced by acetic acid. The duration can be from a few seconds to a few minutes ${ }^{10}$. In patients with colorectal tumors, AA-NBIME enabled a clear visualization of the pit patterns (the shape of the opening of a glandular crypt) and prediction of the histologic features, which technically simplifies the surgical procedure and thus reduces the time ${ }^{11}$. Unfortunately, there are only few published articles concerned with the comparison among NBI, NBIME and AA-NBIME on the diagnosis of small colorectal polyps. In this study, we aimed to compare the diagnostic accuracy among NBI, NBIME and AA-NBIME.

\section{Results}

Clinical characteristics of participants. A total of 261 polyps were identified and analyzed in 122 patients. 2 polyps were excluded due to insufficient enhancement by acetic acid. 85 were men and 37 were women. The mean age of all patients with polyps was 54.2 years (range 43.4-65.0 years). The size of 132 polyps was in 1-5 $\mathrm{mm}$ and 129 in 6-10 $\mathrm{mm} .79$ polyps were found in the location of right colon (cecum, ascending and transverse colon), 119 in the left colon (descending and sigmoid colon) and 63 in the rectum (Table 1).

Comparison between observations and histologic diagnosis under different modalities. Predicted and actual histology for small colorectal polyps were given as follows. For three experts, the number of colorectal tumorous lesions diagnosed by NBI, NBIME and AA-NBIME were 163, 161 and 161, among which 148, 152 and 156 were confirmed by histopathologic assessment. The number of non-neoplastic polyps diagnosed by NBI, NBIME and AA-NBIME were 98, 100 and 100, among which 81, 87 and 91 were confirmed by histopathologic assessment. For three non-experts, the number of colorectal tumorous lesions diagnosed by NBI, NBIME and AA-NBIME were 157, 158 and 165, among which 135, 141 and 151 were confirmed by histopathologic assessment. The number of non-neoplastic polyps diagnosed by NBI, NBIME and AA-NBIME were 104, 103 and 96, among which 74, 79 and 82 were confirmed by histopathologic assessment (Table 2).

Diagnostic accuracy of small colorectal polyps in the expert group. For three experts, as shown in Table 3, AANBIME exhibited a significantly higher specificity, positive predictive value (PPV), negative predictive value $(\mathrm{NPV})$ and accuracy than those of NBI $(P<0.05)$. The diagnostic sensitivity, specificity, negative predictive value (NPV) and accuracy of AA-NBIME were all higher than NBIME. However, no statistically significant differences were found between AA-NBIME and NBIME. Moreover, for NBIME, the diagnostic sensitivity, specificity, positive predictive value (PPV), negative predictive values (NPV) were all significantly higher than those for NBI $(P<0.05)$. The accuracy was also higher than NBI. However, this was only a trend without statistically significant difference between NBIME and NBI (Table 3).

Diagnostic accuracy of small colorectal polyps in the non-expert group. For three non-experts, AA-NBIME had a significantly higher sensitivity, positive predictive value (PPV), negative predictive value (NPV) and accuracy than NBI $(P<0.05)$. AA-NBIME exhibited a significantly higher sensitivity and negative predictive value (NPV) than those of NBIME $(P<0.05)$. The diagnostic specificity, positive predictive value $(\mathrm{PPV})$ and accuracy of AANBIME were all higher than NBIME. However, no statistically significant differences were found between them. What's more, the diagnostic sensitivity, specificity, positive predictive value (PPV) and negative predictive value (NPV) of NBIME were all significantly higher than NBI $(P<0.05)$. The accuracy was also higher than NBI. However, this was only a trend without statistically significant difference between NBIME and NBI (Table 4).

Diagnostic accuracy of small colorectal polyps between expert group and non-expert group among different modalities. In all three modalities, the non-expert group's diagnostic accuracies were 


\begin{tabular}{|l|l|l|}
\hline \multicolumn{2}{|l|}{ Neoplastic, n (\%) } & Non-neoplastic, n (\%) \\
\hline (a) Experts-agreed diagnosis & $148(56.7)$ & $15(5.7)$ \\
\hline NBI & $17(6.5)$ & $81(31.0)$ \\
\hline Neoplastic & $152(58.2)$ & $9(3.4)$ \\
\hline Non-neoplastic & $13(5.0)$ & $87(33.3)$ \\
\hline NBIME & $156(60.0)$ & $5(2.0)$ \\
\hline Neoplastic & $9(3.4)$ & $91(34.9)$ \\
\hline Non-neoplastic & \multicolumn{2}{|l|}{} \\
\hline AA-NBIME & $135(51.7)$ & $22(8.4)$ \\
\hline Neoplastic & $30(11.5)$ & $74(28.4)$ \\
\hline Non-neoplastic & \multicolumn{2}{|l|}{} \\
\hline (b) Non-experts-agreed diagnosis & $17(6.5)$ \\
\hline NBI & $141(54.0)$ & $79(30.3)$ \\
\hline Neoplastic & $24(9.2)$ & $14(5.4)$ \\
\hline Non-neoplastic & $151(57.9)$ & $82(31.4)$ \\
\hline NBIME & $14(5.4)$ & \\
\hline Neoplastic &
\end{tabular}

Table 2. Correlation between (a) "experts-agreed diagnosis", (b) "non-experts-agreed diagnosis" for each modality and histopathologic type.

\begin{tabular}{|l|l|l|l|l|l|l|}
\hline Modality & NBI (95\% CI) & NBIME (95\% CI ) & AA-NBIME (95\% CI) & $\boldsymbol{P}_{\text {NBIvsAA-NBIME }}$ & $\boldsymbol{P}_{\text {NBIvsNBIME }}$ & $\boldsymbol{P}_{\text {NBIMEvsAA-NBIME }}$ \\
\hline Sensitivity & $89.7 \%(85.1-94.3)$ & $92.1 \%(88.0-96.2)$ & $94.5 \%(91.1-98.0)$ & 0.117 & 0.045 & 0.394 \\
\hline Specificity & $84.4 \%(77.1-91.6)$ & $90.6 \%(84.8-96.5)$ & $94.8 \%(90.3-99.2)$ & 0.008 & 0.014 & 0.285 \\
\hline Accuracy & $87.7 \%(83.2-91.2)$ & $91.6 \%(87.5-94.4)$ & $94.6 \%(91.1-96.9)$ & 0.009 & 0.196 & 0.227 \\
\hline PPV & $90.8 \%(86.4-95.2)$ & $94.4 \%(90.9-98.0)$ & $96.9 \%(94.2-99.6)$ & 0.006 & 0.011 & 0.266 \\
\hline NPV & $82.7 \%(75.2-90.1)$ & $87.0 \%(80.4-93.6)$ & $91.0 \%(85.4-96.6)$ & 0.069 & 0.017 & 0.342 \\
\hline
\end{tabular}

Table 3. Diagnostic performance of optical diagnosis of colorectal neoplastic polyps by three modalities for the expert group. $P P V$ positive predictive value, $N P V$ negative predictive value.

\begin{tabular}{|l|l|l|l|l|l|l|}
\hline Modality & NBI (95\% CI) & NBIME (95\% CI ) & AA-NBIME (95\% CI) & $\boldsymbol{P}_{\text {NBIvsAA-NBIME }}$ & $\boldsymbol{P}_{\text {NBIvsNBIME }}$ & $\boldsymbol{P}_{\text {NBIMEvsAA-NBIME }}$ \\
\hline Sensitivity & $81.8 \%(75.9-87.7)$ & $85.5 \%(80.1-90.8)$ & $91.5 \%(87.3-95.8)$ & $<0.001$ & 0.014 & 0.033 \\
\hline Specificity & $77.1 \%(68.7-85.5)$ & $82.3 \%(74.7-89.9)$ & $85.4 \%(78.4-92.5)$ & 0.157 & 0.025 & 0.590 \\
\hline Accuracy & $80.1 \%(74.8-84.5)$ & $84.3 \%(79.4-88.2)$ & $89.3 \%(84.9-92.5)$ & 0.005 & 0.253 & 0.121 \\
\hline PPV & $86.0 \%(80.6-91.4)$ & $89.2 \%(84.4-94.1)$ & $91.5 \%(87.3-95.8)$ & 0.083 & 0.012 & 0.469 \\
\hline NPV & $71.2 \%(62.4-79.9)$ & $76.7 \%(68.5-84.9)$ & $85.4 \%(78.4-92.5)$ & $<0.001$ & 0.004 & 0.029 \\
\hline
\end{tabular}

Table 4. Diagnostic performance of optical diagnosis of colorectal neoplastic polyps by three modalities for the non-expert group. $P P V$ positive predictive value, $N P V$ negative predictive value.

statistically significantly lower compared with the expert group (NBI, $P=0.024$; NBIME, $P=0.011$; AA-NBIME, $P=0.024$ ) (Table 5).

Comparison of the interobserver diagnostic agreement among $M E, N B I M E$, and AA-NBIME. For the expert group, the kappa values, expressed as $95 \%$ confidence interval (CI), for AANBIME, NBIME and ME were 0.962 (0.892-1.032), 0.577 (0.507-0.647), 0.567 (0.497-0.637), respectively, showing "almost perfect" agreement for AA-NBIME, "substantial" agreement for NBIME, and "moderate" agreement for ME. For the non-expert group, the kappa values $(95 \% \mathrm{CI})$ for AA-NBIME, NBIME and ME were $0.818(0.748-0.888), 0.532(0.462-0.602)$ and $0.530(0.460-0.600)$, respectively, showing almost a perfect agreement for AA-NBIME, a moderate agreement not only for NBIME but also for ME (Table 6). 


\begin{tabular}{|l|l|l|l|}
\hline \multirow{2}{*}{ Modality } & \multicolumn{2}{|l|}{ Accuracy } & \multirow{2}{*}{} \\
\cline { 2 - 3 } & Expert group (\%) & Non-expert group (\%) & $\boldsymbol{P}$ \\
\hline NBI & 87.7 & 80.1 & 0.024 \\
\hline NBIME & 91.6 & 84.3 & 0.011 \\
\hline AA-NBIME & 94.6 & 89.3 & 0.024 \\
\hline
\end{tabular}

Table 5. Diagnostic accuracy of small colorectal polyps between the expert group and non-expert group for different modalities.

\begin{tabular}{|l|l|l|}
\hline \multirow{2}{*}{ Modality } & \multicolumn{2}{|l|}{ Kappa value $(\mathbf{9 5} \% \mathbf{C I})$} \\
\cline { 2 - 3 } & Expert group & Non-expert group \\
\hline ME & $0.567(0.497-0.637)$ & $0.530(0.460-0.600)$ \\
\hline NBIME & $0.577(0.507-0.647)$ & $0.532(0.462-0.602)$ \\
\hline AA-NBIME & $0.962(0.892-1.032)$ & $0.818(0.748-0.888)$ \\
\hline
\end{tabular}

Table 6. Kappa statistics of interobserver agreement for diagnosis among three modalities for experts and non-experts.

\begin{tabular}{|l|l|l|}
\hline \multirow{2}{*}{ Modality } & \multicolumn{2}{|l|}{ Image quality scores $(\boldsymbol{t} \pm \boldsymbol{s})$} \\
\cline { 2 - 3 } & Expert group & Non-expert group \\
\hline NBI & $2.69 \pm 0.85^{\star * *}$ & $2.32 \pm 1.15^{\star * *}$ \\
\hline NBIME & $2.96 \pm 0.77^{* * *}$ & $3.23 \pm 0.89^{* * *}$ \\
\hline AA-NBIME & $3.20 \pm 0.66$ & $3.39 \pm 0.71$ \\
\hline
\end{tabular}

Table 7. Comparison of the image quality among NBI, NBIME, and AA-NBIME. ${ }^{* *}$ AA-NBIME, NBIME versus NBI $P<0.001,{ }^{* * *}$ AA-NBIME versus NBIME $P<0.001$.

Comparison of the image quality among NBI, NBIME, and AA-NBIME. With higher values indicating better quality, the scores were: For expert group, NBI, $2.69 \pm 0.85$, NBIME, $2.96 \pm 0.77$, AA-NBIME, $3.20 \pm 0.66$. For non-expert group, NBI, $2.32 \pm 1.15$, NBIME, $3.23 \pm 0.89$, AA-NBIME, $3.39 \pm 0.71$. The average scores (experts and non-experts) of images acquired using AA-NBIME and NBIME were significantly higher than those acquired using NBI $(P<0.001)$. The average scores (experts and non-experts) of images acquired using AA-NBIME were significantly higher than those acquired using NBIME $(P<0.001)$ (Table 7$)$.

\section{Discussion}

It is widely accepted that most colorectal carcinomas appear to arise from adenomas ${ }^{2}$, and the removal of adenomatous polyps by colonoscope has already result in significant reductions in the incidence of $\mathrm{CRC}^{3}$. It is generally agreed that regular colonoscopy surveillance is necessary after these polypectomies. It has been reported that more than $90 \%$ of colonoscopy-identified polyps were small polyps $(6-9 \mathrm{~mm})$ or diminutive polyps $(<5 \mathrm{~mm})$, and diminutive polyps are dominant ${ }^{12,13}$. The majority of small colon polyps are non-neoplastic, and most of which are hyperplastic in nature ${ }^{12,13}$. Therefore, these polypectomies are unnecessary to perform, posing risks of bleeding and perforation ${ }^{14,15}$. Real-time optical diagnosis of recto-sigmoid small polyps would allow hyperplastic polyps to be left in situ and adenomatous polyps to be removed without histopathology ${ }^{16}$.

NBI is a technology that uses 3 optical filters for red-blue-green sequential illumination and narrows the bandwidth of the spectral transmittance supporting the imaging of mucosal PPs and capillary vessels ${ }^{5}$. NBI can be switched on and off with a button on the endoscope and save the additional cost for dye spraying. NBI in combination with magnifying endoscopy is a promising tool for superior detection of mucosal PP and microvasculature details $^{10,17-20}$, in order to differentiate non-neoplastic from neoplastic colorectal polyps ${ }^{5}$. Iwatate et al. ${ }^{21}$ reported that magnifying endoscopy could improve the performance of NBI in distinguishing neoplastic from non-neoplastic colorectal lesions according to NICE classification. We did not observe any significant benefit of NBIME over NBI for improving the diagnostic accuracy of small colorectal polyps; however, diagnostic accuracy and image quality of NBIME were better than those of NBI.

Acetic acid is a hydrophilic organic acid. It can infiltrate the crypt easily by its small molecular weight, quickly discolor the intervening part between crypts and the marginal crypt epithelium to enable the good visualization of pits ${ }^{11}$. It was reported that magnification endoscopy with acetic acid enhancement and narrow-band imaging is useful for visualizing mucosal microstructure patterns of colorectal polyps.

Magnification endoscopy with crystal violet staining enables clear visualization of the PPs of the colorectal polyps, and helps to differentiate neoplastic from non-neoplastic lesions ${ }^{22-24}$. Kudo et al. ${ }^{25}$ classified different 
morphological features into six PPs based on this techque (Type I, Type II, Type IIIS, Type IIIL, Type IV, Type V), with types I and II regarded as non-polyps, types III (including IIIL and IIIs), IV considered to be adenoma and type $\mathrm{V}$ classified as adenocarcinoma ${ }^{26}$. Despite having the merit of effectively evaluating colorectal polyps, this technique requires the use of $0.05 \%$ crystal violet spraying and is technically hard for endoscopists. It's reported that AA-NBIME diagnosis was not inferior that of magnification endoscopy with crystal violet staining in the diagnostic accuracy of colorectal polyps. In addition, it is easier to operate ${ }^{27}$. This study finds that for experts and non-experts, the diagnostic accuracy of AA-NBIME were all significantly higher than NBI. AA-NBIME didn't show a statistically significantly higher diagnostic accuracy compared to NBIME, however, the performance of AA-NBIME was better than that of NBIME in terms of diagnostic accuracy.

We also believe that, of all three modalities, the performance of expert group was better than that of the nonexpert group in terms of diagnostic accuracy. Rogart et al. ${ }^{24}$ reported that diagnostic accuracy using NBI significantly improved with the increasing experience level of endoscopists. They also pointed out that the experience from diagnosing nearly 130 polyps is essential for basic competency. This finding emphasized the importance of practice and experience of endoscopists, which agreed with our results.

In addition, acetic acid removes the mucus adherent to the colorectal polyps through breaking the disulfide bonds of mucus, enabling the better visualization of the lesions. In this study, the average scores (experts and non-experts) of images acquired using AA-NBIME were significantly higher than those acquired using NBIME and NBI.

AA-NBIME is a promising tool to clearly visualize the mucosal PP of colorectal polyps for better differentiating neoplastic polyps from non-neoplastic ones. In conclusion, for experts and non-experts, AA-NBIME showed statistically significantly higher diagnostic accuracy for small colorectal polyps.

There were also some limitations in this study. This study was performed at only two centers. Adequate evaluation of the efficacy of AA-NBIME needs a multicenter trial with more patients. What's more, to maintain the image quality, all the endoscopic images were taken by a single experienced endoscopist. The procedure of magnification endoscopic is technically hard to non-experts, which might limit the applicability of AA-NBIME. We also acknowledge that although we distributed all the images in a random order intermixed with other lesions to the six endoscopists, the possibility that one endoscopist could simultaneously recognize the three modalities of the same lesion cannot be ruled out, which would interfere with his diagnosis of the lesion.

\section{Methods}

Participants. Patients scheduled for performing polypectomies $(<1 \mathrm{~cm})$ and agreed with ME at Jiangsu Province Hospital and Jingjiang People's Hospital between May 2017 and January 2018 were consecutively enrolled in this study. We excluded patients from enrolment who had coagulopathy or a platelet count less than $50,000 / \mathrm{mm}^{3}$ or lesions covered with adherent mucus or blood, insufficiently enhanced by the acetic acid. The study was performed in accordance with the Declaration of Helsinki and was approved by the Ethics Committee of the Jingjiang People's Hospital, Jingjiang, Jiangsu, China and Jiangsu Province Hospital, Nanjing, Jiangsu, China. All patients signed written informed consent for every procedure.

\section{Endoscopic procedure and therapy.}

1. Bowel preparation: All patients were prepared for colonoscopy with $2 \mathrm{~L}$ of polyethylene glycol-electrolyte solution and dimethicone administered on the morning of the examination.

2. Endoscopic Procedure: A conventional colonoscope was used first for routine colonoscopy. When the colonoscope arrived to ileocecus, reinspect the colon during withdrawal from the cecum. Once a polyp was detected, surface mucus was washed away with lukewarm water, and optical diagnosis was made using magnifying Olympus CF-H260Z colonoscopes. Endoscopic images were taken in the following order: Narrow-band imaging (NBI), Magnification endoscopy with narrow-band imaging (NBIME) and Magnification endoscopy with acetic acid (1.5\%)-enhanced NBI (AA-NBIME). For AA-NBIME, 5-10 $\mathrm{ml}$ of 1.5\% acetic acid solution was dripped onto the polyps using a special tube, which were inserted from the forceps channel of the endoscope and the microstructure of the same areas that had been observed by NBI and NBIME were photographed with NBIME under acetic acid-enhanced conditions. Diagnosis was made using NBIME under the enhancement of acetic acid. Endoscopic images were taken before and 15-30 s after spraying under the condition of full air inflation ${ }^{28}$. All endoscopic images were taken by a single expert endoscopist (Sha) under different modalities, who had experience of over 2000 cases of magnification colonoscopy with NBI.

3. Therapy: All polyps were removed by biopsy forceps or Endoscopic Mucosal Resection (EMR) and sent for histological assessment.

Assessment of endoscopic images. According to NICE and Kudo PP classifications, all of the endoscopic images of NBI, NBIME and AA-NBIME were displayed for each modality in a random order, and NBIME and AA-NBIME images were displayed alone, without the risk of bias caused by corresponding NBI image.

The endoscopic images of each modality were independently reviewed by six endoscopists (three experts and three non-experts), who were all blinded to the final histological diagnosis. The three experts were well versed and had 5 years' experience in narrow-band imaging and magnifying endoscopy in the colorectum and the three non-experts were proficient in conventional colonoscopy, while without any experience in narrow-band imaging or magnifying endoscopy. Images taken under NBI and NBIME were assessed according to NICE classification, while those taken under AA-NBIME were assessed according to Kudo PP classification. Diagnostic accuracy was compared among three modalities based on Histopathological results. Before starting the study, the non-expert group completed a training session on NICE and Kudo PP classifications. In both groups, the accuracy of the 

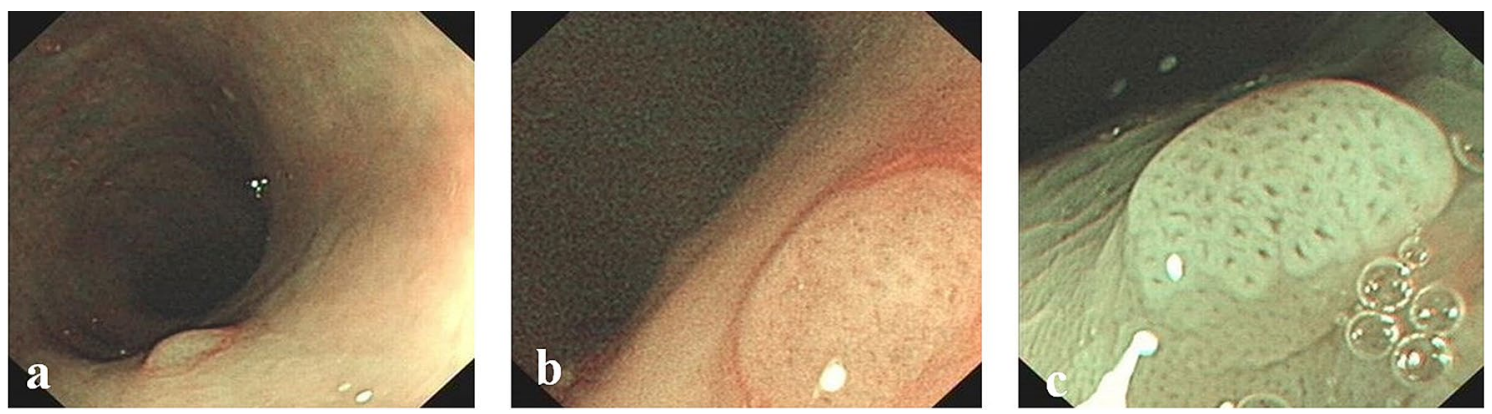

Figure 1. Surface patterns of non-neoplastic polyps viewed by different modalities: (a) NBI; (b) NBIME; (c) AA-NBIME.
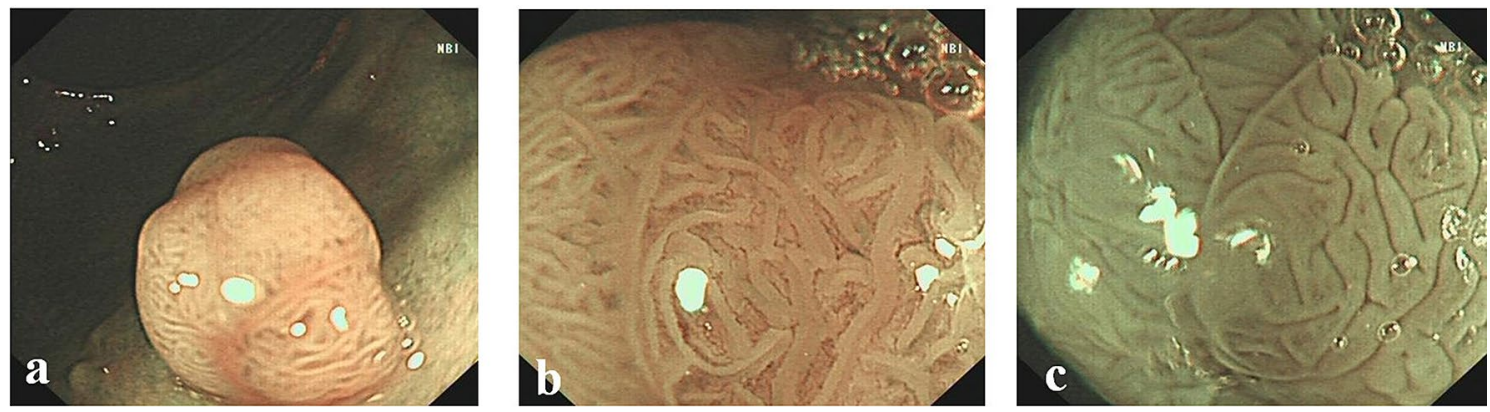

Figure 2. Surface patterns of neoplastic polyps viewed by different modalities: (a) NBI; (b) NBIME; (c) AA-NBIME.

modality was included in the study only when two or three members had the same idea on the classification of the lesion. The NBI International Colorectal Endoscopic (NICE) classification was as follows: Type 1: Color: Same or lighter than background; Vessels: None, or isolated lacy vessels coursing across the lesion; Surface pattern: Dark or white spots of uniform size, or homogeneous absence of pattern (Fig. 1); Type 2: Color: Browner relative to background (verify color arises from vessels); Vessels: Brown vessels surrounding white structures; Surface pattern: Oval, tubular, or branched white structures surrounded by brown vessels (Fig. 2). Type 1 was most likely to be hyperplastic, while Type 2 was most likely to be adenoma ${ }^{6}$. The Kudo PP classification was as follows: Type I: round pits ; Type II: stellar or papillary pits ; Type III L: large tubular or roundish pits ; Type III S: small tubular or roundish pits ; Type IV: branch-like or gyrus-like pits ; Type V: non-structural pits ${ }^{11}$. Types I and II were classified as non - neoplastic, as shown in Fig. 1, whereas types III, IV and V were regarded as neoplastic lesions, as shown in Fig. 2. Image quality classification was as follows: score 1: unobservable; Score 2: blurred; score 3: less clear; score 4: clear.

To verify the clinical feasibility and availability of each diagnostic modality, kappa statistics were applied for analyzing an interobserver diagnostic agreement among each modality for the expert and non-expert groups.

Histopathological assessment. All polyps were immediately fixed in $10 \%$ formalin and then standard H\&E staining was performed. A single expert pathologist, who was blinded to the endoscopic assessment, judged the histologic characteristics of the specimens.

Statistical analysis. The statistical analysis was performed using the SPSS for Windows Version 16.0 statistical software package. Comparisons for sensitivity, specificity, and accuracy among the three modalities were tested by paired Chi-square method. The differences in positive predictive value and negative predictive value of the three modalities were compared by the method proposed by Kosinski ${ }^{29}$. The image definition score is shown as $\mathrm{x} \pm \mathrm{s}$ and one-way analysis of variance was applied in order to compare the scores among three modalities. $P$ value of $<0.05$ was considered to be statistically significant.

With kappa statistics, we also analyzed the interobserver diagnostic agreement for the expert and non-expert groups. Data are expressed as point estimates of kappa with 95\% CI. In theory, perfect disagreement has a kappa value of -1.0 , and a kappa value of 1.0 is considered perfect agreement. A value of 0 indicates an agreement by chance alone. According to the Landis and Koch scale, kappa values are graded as follows: $0.01-0.2$ slight, 0.21-0.4 fair, 0.41-0.6 moderate, 0.61-0.8 substantial, and 0.81-1.0 almost perfect ${ }^{30,31}$.

Received: 29 May 2019; Accepted: 23 November 2020

Published online: 16 December 2020 


\section{References}

1. Okada, T. et al. International collaboration between Japan and Chile to improve detection rates in colorectal cancer screening. Cancer 122, 71-77 (2016).

2. Vogelstein, B. et al. Genetic alterations during colorectal-tumor development. N. Engl. J. Med. 319, 525-532 (1988).

3. Brenner, H., Stock, C. \& Hoffmeister, M. Effect of screening sigmoidoscopy and screening colonoscopy on colorectal cancer incidence and mortality: Systematic review and meta-analysis of randomised controlled trials and observational studies. BMJ 348, g2467 (2014).

4. Wang, P. et al. Outcome of a novel modified endoscopic papillectomy for duodenal major papilla adenoma. Surg. Endosc. https:// doi.org/10.1007/s00464-020-07715-0 (2020).

5. An, J. K. et al. Marginal turbid band and light blue crest, signs observed in magnifying narrow-band imaging endoscopy, are indicative of gastric intestinal metaplasia. BMC Gastroenterol. 12, 169 (2012).

6. Hewett, D. G. et al. Validation of a simple classification system for endoscopic diagnosis of small colorectal polyps using narrowband imaging. Gastroenterology 143, 599-607.e1 (2012).

7. Nonaka, K., Arai, S., Ban, S. \& Kitada, H. Prospective study of the evaluation of the usefulness of tumor typing by narrow band imaging for the differential diagnosis of gastric adenoma and well-differentiated adenocarcinoma. Dig Endosc. 23, 146-152 (2011).

8. Nakayoshi, T. et al. Magnifying endoscopy combined with narrow band imaging system for early gastric cancer: Correlation of vascular pattern with histopathology (including video). Endoscopy 36, 1080-1084 (2004).

9. Kadowaki, S. et al. Ease of early gastric cancer demarcation recognition: A comparison of four magnifying endoscopy methods. J. Gastroenterol. Hepatol. 24, 1625-1630 (2009).

10. Yagi, K., Aruga, Y., Nakamura, A., Sekine, A. \& Umezu, H. The study of dynamic chemical magnifying endoscopy in gastric neoplasia. Gastrointest. Endosc. 62, 963-969 (2005).

11. Shibagaki, K. et al. Magnification endoscopy with acetic acid enhancement and a narrow-band imaging system for pit pattern diagnosis of colorectal neoplasms. J. Clin. Gastroenterol. 49, 306-312 (2015).

12. Lieberman, D., Moravec, M., Holub, J., Michaels, L. \& Eisen, G. Polyp size and advanced histology in patients undergoing colonoscopy screening: implications for CT colonography. Gastroenterology 135, 1100-1105 (2008).

13. Rex, D. K., Overhiser, A. J., Chen, S. C., Cummings, O. W. \& Ulbright, T. M. Estimation of impact of American College of Radiology recommendations on CT colonography reporting for resection of high-risk adenoma findings. Am. J. Gastroenterol. 104, 149-153 (2009).

14. Togashi, K. et al. Efficacy of magnifying endoscopy in the differential diagnosis of neoplastic and non-neoplastic polyps of the large bowel. Dis. Colon Rectum 42, 1602-1608 (1999).

15. Su, M.-Y. et al. Magnifying endoscopy with indigo carmine contrast for differential diagnosis of neoplastic and nonneoplastic colonic polyps. Dig. Dis. Sci. 49, 1123-1127 (2004).

16. Rees, C. J. et al. Narrow band imaging optical diagnosis of small colorectal polyps in routine clinical practice: The Detect Inspect Characterise Resect and Discard 2 (DISCARD 2) study. Gut 66, 887-895 (2017).

17. Tischendorf, J. J. W. et al. Value of magnifying chromoendoscopy and narrow band imaging (NBI) in classifying colorectal polyps: A prospective controlled study. Endoscopy 39, 1092-1096 (2007).

18. Hirata, M. et al. Magnifying endoscopy with narrow band imaging for diagnosis of colorectal tumors. Gastrointest. Endosc. 65, 988-995 (2007).

19. Kanao, H. et al. Narrow-band imaging magnification predicts the histology and invasion depth of colorectal tumors. Gastrointest. Endosc. 69, 631-636 (2009).

20. Wada, Y. et al. Diagnosis of colorectal lesions with the magnifying narrow-band imaging system. Gastrointest. Endosc. 70, 522-531 (2009).

21. Iwatate, M. et al. The addition of high magnifying endoscopy improves rates of high confidence optical diagnosis of colorectal polyps. Endosc. Int. Open 3, E140-E145 (2015).

22. Hurlstone, D. P., Cross, S. S., Slater, R., Sanders, D. S. \& Brown, S. Detecting diminutive colorectal lesions at colonoscopy: A randomised controlled trial of pan-colonic versus targeted chromoscopy. Gut 53, 376-380 (2004).

23. Fujii, T. et al. Chromoscopy during colonoscopy. Endoscopy 33, 1036-1041 (2001).

24. Rogart, J. N. et al. Narrow-band imaging without high magnification to differentiate polyps during real-time colonoscopy: Improvement with experience. Gastrointest. Endosc. 68, 1136-1145 (2008).

25. Kudo, S. et al. Colonoscopic diagnosis and management of nonpolypoid early colorectal cancer. World J. Surg. 24, 1081-1090 (2000).

26. Kudo, S. et al. Diagnosis of colorectal tumorous lesions by magnifying endoscopy. Gastrointest. Endosc. 44, 8-14 (1996).

27. Lambert, R., Rey, J. F. \& Sankaranarayanan, R. Magnification and chromoscopy with the acetic acid test. Endoscopy 35, 437-445 (2003).

28. Kim, J.-H. et al. Importance of the surrounding colonic mucosa in distinguishing between hyperplastic and adenomatous polyps during acetic acid chromoendoscopy. World J. Gastroenterol. 14, 1903-1907 (2008).

29. Kosinski, A. S. A weighted generalized score statistic for comparison of predictive values of diagnostic tests. Stat Med. 32(6), 964-77. https://doi.org/10.1002/sim.5587 (2013).

30. Landis, J. R. \& Koch, G. G. The measurement of observer agreement for categorical data. Biometrics 33, 159-174 (1977).

31. Bland, J. M. \& Altman, D. G. Statistical methods for assessing agreement between two methods of clinical measurement. Lancet 1, 307-310 (1986)

\section{Acknowledgements}

Funding for the present study was provided by Jingjiang City key talent research project. The authors appreciate the expert technical assistance from the Department of Gastroenterology of the First Affiliated Hospital of Nanjing Medical University. We also thank Chunyan Wu and Yu Zheng for participating in data interpretation and graphic drawing.

\section{Author contributions}

F.G. and Y.J. designed the study. J.S., P.W., N.S. and H.A.Z. contributed equally to experiments and the writing of the manuscript. A.P.Y., L.C. and X.L.L. were responsible for interpreting the data. C.L., Y.M.Q. and Z.W.G. were responsible for the graphics of the manuscript. All authors read and approved the final manuscript.

\section{Competing interests}

The authors declare no competing interests. 


\section{Additional information}

Correspondence and requests for materials should be addressed to Y.J. or F.G.

Reprints and permissions information is available at www.nature.com/reprints.

Publisher's note Springer Nature remains neutral with regard to jurisdictional claims in published maps and institutional affiliations.

(c) (1) Open Access This article is licensed under a Creative Commons Attribution 4.0 International License, which permits use, sharing, adaptation, distribution and reproduction in any medium or format, as long as you give appropriate credit to the original author(s) and the source, provide a link to the Creative Commons licence, and indicate if changes were made. The images or other third party material in this article are included in the article's Creative Commons licence, unless indicated otherwise in a credit line to the material. If material is not included in the article's Creative Commons licence and your intended use is not permitted by statutory regulation or exceeds the permitted use, you will need to obtain permission directly from the copyright holder. To view a copy of this licence, visit http://creativecommons.org/licenses/by/4.0/.

(c) The Author(s) 2020 